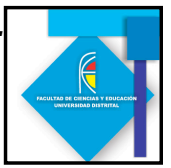

\title{
CREACIÓN LITERARIA EN EL MARCO DE LA ENSEÑANZA DE LA FÍSICA CON ESTUDIANTES DE GRADO OCTAVO DE BACHILLERATO.
}

\section{CREATIVEWRITING WITHIN THE PHYSICS CLASS WITH EIGHTH GRADE STUDENTS}

Fabián Ricardo Carvajal Córdoba ${ }^{1}$

\section{Resumen}

La enseñanza de la física debe articularse con otras áreas del conocimiento y por tanto, la práctica del docente debe reflejar ese trabajo invitando a conocer la física, desde otras perspectivas, mediante la escritura, la lectura y la creatividad. De acuerdo con lo anterior, en esta propuesta se plantea plasmar la relación de la creación literaria con la física mediante un ejercicio con estudiantes de grado octavo a partir de algunos postulados básicos del movimiento de enseñanza escritura transcurricular. Los estudiantes crean un poema, un cuento o un escrito libre acerca de los temas escogidos (los estados de la materia, y termodinámica) y sus creaciones serán puestas en un poster que exponga algunos trabajos junto con las principales componentes que los estudiantes muestran a la hora de realizar este tipo de ejercicios. El trabajo se plantea con estudiantes de grado octavo ya que es una etapa educativa en la que la imaginación y la creatividad caracterizan el trabajo de cada día.

Palabras clave: enseñanza, física, literatura, escrituratranscurricular.

\section{Abstract}

The teaching of Physics must be related with other subjects. Therefore, the teacher's performance should lead the knowledge of Physics from other perspectives, such as reading, writing and creative thinking. According to this, it has been proposed to establish the relationship between Creative Writing and Physics through an exercise with Eighth Grade students following the basic postulates of the Writing Across Curriculum movement.

Keywords: Teaching, Physics, Literature, Writing Across Curriculum.

\footnotetext{
${ }^{1}$ Universidad Pedagógica Nacional, frcc622@hotmail.com
} 


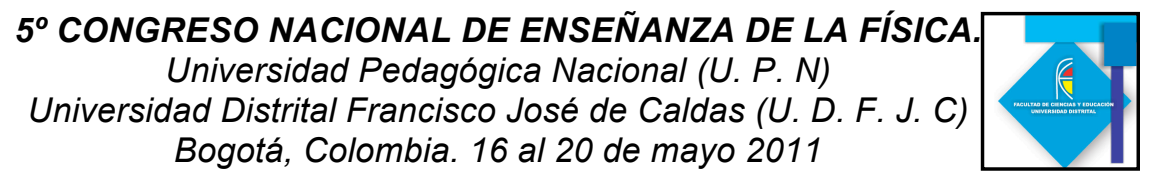

\section{Introducción}

Además de ser bienvenido con una gran celebración, el inicio del siglo XXI presenta un cambio en el modo como las personas se relacionan con la tecnología, en especial jóvenes estudiantes que todavía no acaban su formación en el colegio. Al pasar el tiempo ha ido aumentando tanto el número de jóvenes que acuden a todo tipo de tecnologías como los mismos avances que traen consigo un futuro prometedor para las industrias que promocionan estos artículos y servicios. Pero para los jóvenes, visto desde un punto de vista pedagógico, la inmersión en un mundo totalmente tecnológico ha traído consecuencias que se ven reflejadas en la enseñanza-aprendizaje de las humanidades y las ciencias por ejemplo. La escritura (manual) como ejercicio en el aula se está perdiendo, los jóvenes cada vez escriben menos. De la mano, la lectura de libros es vista con menos interés cada vez. En resumen, los estudiantes no utilizan un bolígrafo o un lápiz para escribir sobre una hoja en blanco, y no capturan un libro entre sus manos para leer, a menos que sea virtual. La consecuencia es que la imaginación se está limitando y por ello la creación literaria aparece cada vez menos en las áreas de conocimiento.

La enseñanza de la física no puede ser ajena a este tipo de situaciones, y por ello la interdisciplinariedad de áreas de conocimiento debe estar constantemente presente en las posibles soluciones a los problemas de enseñanza, por ejemplo la física. Sanmartí (1995), Jorba, Gomez y Prat (1998) entre otros, citados por Daniel Cassany (1999), indican que todo maestro es de lengua, o que la lengua es una materia instrumental en la enseñanza. Esto resume la idea de abrir el camino entre Lengua y otras disciplinas curriculares.

Con los nuevos movimientos de enseñanza, tal comoWritingAcrosstheCurriculum; WAC (escritura transcurricular), la enseñanza de la física ha adquirido más importancia, y se pretende implicar la composición, la redacción y la escritura misma en la enseñanza de temas como el movimiento, la energía, los estados de la materia y la ubicación espacial.

En el bachillerato los estudiantes de grado octavo asocian algunas de las ideas acerca de fenómenos físicos que hacen parte de su cotidianidad. Los estados de la materia y los procesos que tienen que ver con cambios de temperatura y energía, son ideales para abordar un tipo de clase en donde se haga uso del experimento. Al despertar el interés de los estudiantes y comenzar a realizar informes de laboratorio, elaborados en forma, la escritura entra en juego y parece el momento indicado de abordar la creación literaria, desde la escritura transcurricular, como forma alterna de plasmar el conocimiento adquirido por los estudiantes.

\section{Desarrollo de la propuesta: escritura transcurricular en el aula}

Presentamos un fragmento del libro de Cassany (1999), en el que indica los principales supuestos acerca del desarrollo del movimiento de promoción de escritura llamado escritura transcurrricular.

"actividades que favorecen la construcción de conocimiento: 


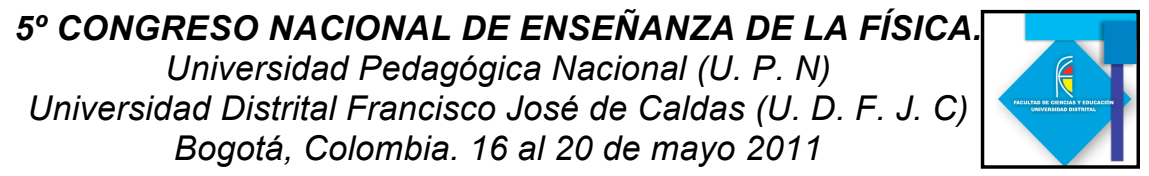

Activan los conocimientos previos del estudiante. Hace emerger ideas y opiniones.

Plantean situaciones comunicativas.

Contrastar distintos estadios de elaboración de sus opiniones propias, y compararlas con los compañeros y el docente."(Cassany, 1999)

La propuesta en el aula se plantea, entonces desde la perspectiva del movimiento de escritura transcurricular, con estudiantes de grado octavo grado de bachillerato. Por lo tanto, las actividades a llevar a cabo se plasman como sigue según el autor Suton (1996), citado en el libro de (Cassany, 1999).

"Desde la didáctica de las ciencias se defiende la importancia de utilizar un lenguaje personalizado, subjetivo, e interpretativo para enseñar-aprender ciencia"

En este orden de ideas, la actividad dentro del aula, desde los temas los estados de la materia, y termodinámica, desarrollará las funciones de la composición en la escritura según Fulwiler (1987) citado en el libro de (Cassany, 1999).

"funciones epistémicas de la composición:

Actividades de escritura libre o exploratoria (free writing). El estudiante redacta espontáneamente sobre temas concretos, para descubrir sus opiniones."

A modo de ejemplo se plasma uno de los trabajos realizados, con la modalidad de composición libre. En este caso el estudiante ha elegido un simple dibujo donde plasma lo aprendido después de una clase acerca de la presión de los líquidos.

La imagen 1 es uno de los muchos ejemplos que se plasmarán en el poster a realizar. Estudiantes de grado octavo, desde la asignatura de física realizarán un escrito, un poema, un cuento o una historieta. En este caso, un estudiante hace un dibujo de lo realizado después de haber culminado el tema de Presión en la clase de física.

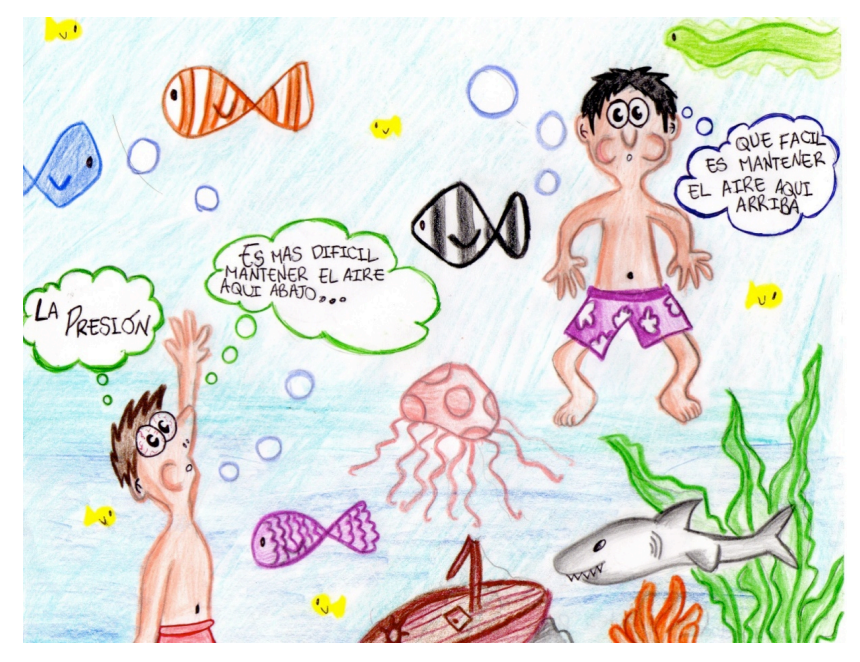

Imagen 1. Composición estudiante acerca de la presión en líquidos. 


\section{Conclusiones}

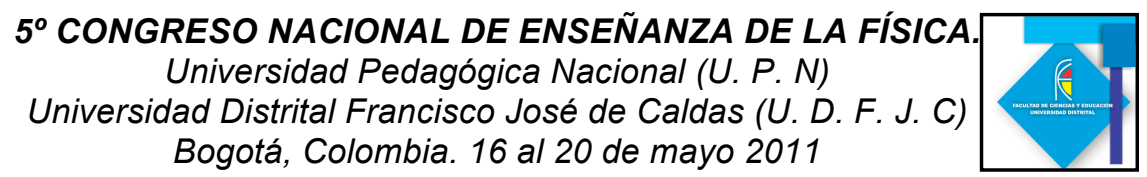

El trabajo realizado en el aula muestra claramente la posición desde el movimiento escritura transcurricularen la que se resalta el conocimiento previo de los estudiantes a la hora de hacer composiciones de este tipo. El lenguaje que describen los estudiantes es el cotidiano, y de allí se puede interpretar el entendimiento y el conocimiento adquirido. En la figura 1 el estudiante claramente esta relacionando la presión con un esfuerzo, y entiende la relación que existe la presión dentro de un fluido con pesos específicos diferentes (agua y aire).

El estudiante que escribe eficazmente, con placer y orgullo, es cada día más valioso, en un mundo en el que es habitual la comunicación entre lugares y culturas. (Cassany, 1999). Se muestra de esta forma la importancia que se debe recalcar a la hora de enseñar ciencias, en donde la escritura juega un papel muy importante, ya que la enseñanza de la física no está guiada a crear científicos ni mucho menos, sino personas con un sentido comunicativo dentro de la sociedad que cada vez se acelera con tecnologías de la comunicación.

\section{Referencias Bibliográficas}

\section{Sitios Web}

books.google.com.co. (s.f.). Google Books. Recuperado el 24 de Marzo de 2011, dehttp://books.google.com.co/books?id=Ty6Ul3MFZe8C\&pg=PA196\&lpg=PA196\&d $\mathrm{q}=$ escritura+transcurricular\&source=bl\&ots=IVTwGBqB_C\&sig=sQmf-

1xOv1HE1BaQV2tZSt-atMc\&hl=es-

419\&ei=ff6LTaD0AcKW0QGBosCmCw\&sa=X\&oi=book_result\&ct=result\&resnum =1 \&ved $=0$ CBCQ6AEwAA\#v=onepage\&

\section{$\underline{\text { Libros }}$}

Cassany, D. (1999). Construir la escritura. Barcelona: Ediciones Paidós Ibérica S.A. 